\title{
Hollow medium-entropy alloy nanolattices with ultrahigh energy absorption and resilience
}

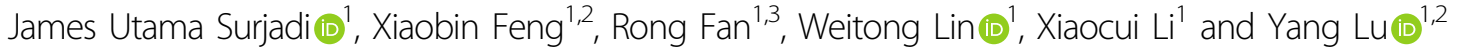

\begin{abstract}
Hollow micro/nanolattices have emerged in recent years as a premium solution compared to conventional foams or aerogels for mechanically robust lightweight structures. However, existing hollow metallic micro/nanolattices often cannot exhibit high toughness due to the intrinsic brittleness from localized strut fractures, limiting their broad applications. Here, we report the development of hollow CoCrNi medium-entropy alloy (MEA) nanolattices, which exhibit high specific energy absorption (up to $25 \mathrm{~J} \mathrm{~g}^{-1}$ ) and resilience (over 90\% recoverability) by leveraging sizeinduced ductility and rationally engineered MEA microstructural defects. This strategy provides a pathway for the development of ultralight, damage-resistant metallic metamaterials for a myriad of structural and functional applications.
\end{abstract}

\section{Introduction}

In the past decade, micro/nanoarchitected materials have proven their potential to overcome canonical couplings between mechanical properties (e.g., strength-density) through the combined benefits of rationally designed cellular topology and size effects ${ }^{1-11}$. Compared to stochastic cellular materials, the ordered and periodic arrangement of internal pores allows micro/ nanolattices to exhibit superior mechanical properties, while bringing the dimensions of a material down to the submicron length scale could unlock unique properties that are generally lost in bulk materials ${ }^{8-11}$. In particular, hollow micro/nanolattices provide the only solution to obtain extremely lightweight materials while simultaneously allowing for high recoverability and specific strength to be achieved. These properties are attributed to the multistage weight reduction resulting from the hollow strut cellular topology as well as the prominence of the

Correspondence: Yang Lu (yanglu@cityu.edu.hk)

${ }^{1}$ Department of Mechanical Engineering, City University of Hong Kong, Kowloon, Hong Kong SAR, China

${ }^{2}$ CityU-Xidian Joint Laboratory of Micro/Nano-Manufacturing \& NanoManufacturing Laboratory (NML), Shenzhen Research Institute of City

University of Hong Kong, 518057 Shenzhen, China

Full list of author information is available at the end of the article

These authors contributed equally: James Utama Surjadi, Xiaobin Feng size effect in influencing the mechanical properties and behavior of nanoscale materials. Among these materials, hollow metallic lattices possess immense potential to achieve simultaneous high strength and ductility ${ }^{1-3}$. Unfortunately, despite their high strength/stiffness, existing hollow metallic lattices possess low toughness, and the inherently brittle nature of their constituents induces catastrophic or localized fractures in high-stress regions (i.e., nodes). This phenomenon has also consistently been observed in other reported solid or coreshell lattices, which is detrimental for practical applications because it significantly degrades their mechanical robustness ${ }^{6,12-14}$.

Recently, owing to their tunable composition and unique microstructures ${ }^{15-18}$, the incorporation of multicomponent alloys, such as high- and medium-entropy alloys (HEAs/MEAs) with micro/nanolattices, into a composite configuration ${ }^{19-21}$ has been proposed, and their potential superiority over conventional metals/alloys in terms of their tunable mechanical (and functional) properties has been demonstrated. Nevertheless, the creation of pure HEA/MEA lattices with hollow struts has never been reported and is challenging but crucial to fully harness their potential for engineering applications (for instance, under extreme temperature environments at 
high/low temperatures ${ }^{22-24}$ or to provide an enhanced surface-to-volume ratio in catalysis ${ }^{25,26}$ ). Herein, we fabricated, for the first time, hollow CoCrNi MEA nanolattices that can exhibit ultrahigh energy absorption and resilience upon repeated loading due to the induced damage resistance derived from the strong and ductile nanostructured MEA film. Combining the benefits of hollow nanoarchitectures with the vast compositional space of HEAs/MEAs presents a new route for the development of lightweight, mechanically robust, and versatile nanoarchitected metamaterials for structural and functional applications ${ }^{25-27}$.

\section{Materials and methods}

\section{Fabrication of hollow MEA nanolattices}

Stretching-dominated octet lattices were synthesized by employing an additive manufacturing technique based on two-photon lithography (Nanoscribe GmbH). The polymer lattices were fabricated using an acrylatebased UV photosensitive resin (IP-L 780). DC magnetron sputtering was subsequently used to conformally coat a thin MEA film onto the polymer scaffold at $5 \times$ $10^{-6} \mathrm{~Pa}$. The argon flow for ignition was set to 10 standard cubic centimeters per minute $(\mathrm{sccm})$, while the total argon flow rate was fixed at $10 \mathrm{sccm}$ for $120 \mathrm{~s}$. The DC power used was $350 \mathrm{~W}$, and the substrate was rotated at a rate of 30 r.p.m. $\mathrm{min}^{-1}$ to ensure uniform film deposition. Subsequently, the internal polymer core of the MEA-coated nanolattice was exposed via focused ion beam (FIB) milling and removed via plasma ashing (Diener Zepto) for $12 \mathrm{~h}$ at $100 \mathrm{~W}$.

\section{Microstructural and compositional characterization}

The film thickness, microstructure, and composition of the nanolattices and reference MEA film were observed by using field emission scanning electron microscopy (FESEM, FEI Quanta FEG450) equipped with energy dispersive X-ray spectroscopy (EDX, Oxford), as well as transmission electron microscopy (TEM, JEOL JEM 2100) equipped with selected area electron diffraction (SAED), where the TEM samples were prepared via ion milling with liquid nitrogen cooling to prevent microstructural changes such as phase transitions. The grain sizes and atomic distribution in the MEA film were investigated via FIB (FEI Scios DualBeam) milling and 3D atomic probe tomography (APT, LEAP 5000XR).

\section{In situ mechanical testing}

The experimental setup for the in situ uniaxial compression tests on the MEA hollow nanolattices, which were performed at room temperature, included a Hysitron PI85 SEM Picoindenter (Bruker) to investigate the mechanical properties and observe the deformation behavior of the lattices. Uniaxial compression tests were conducted on the MEA nanolattices at a prescribed strain rate of $10^{-3} \mathrm{~s}^{-1}$.

\section{Results and discussion \\ Hierarchical structure}

The CoCrNi MEA hollow octet nanolattices were initially prepared by fabrication of polymer nanolattice templates via two-photon lithography direct laser writing (Fig. 1a) followed by magnetron sputtering with a high deposition rate to facilitate the deposition of MEA films with dense nanotwins and stacking faults (SFs) (Fig. 1b). The directionality of sputtering has previously led to nonuniform walls in hollow lattices, generating additional stress concentrations that reduce their mechanical properties. Therefore, the elliptical geometry of the strut cross-section in this work was designed to optimize the uniformity of the deposited metal film (Fig. S1). A FIB was employed to expose the polymer core inside, which was subsequently removed via plasma ashing (Fig. 1c) to ultimately produce hollow MEA nanolattices (Fig. 1d) ${ }^{28}$. Figure $1 \mathrm{e}-\mathrm{h}$ depicts the hierarchical structure of the as-fabricated hollow CoCrNi MEA nanolattice, showing critical feature sizes ranging from tens of micrometers (whole lattice) down to hundreds of nanometers (strut size) and to a few nanometers (planar defects in metal film). Compositional analysis of the deposited MEA film showing a homogenous elemental distribution is shown in Fig. S2.

\section{Mechanical testing}

Figure $2 \mathrm{a}$ and $\mathrm{b}$ shows a series of real-time images depicting the deformation behavior of a thin-walled (i.e., $30 \mathrm{~nm}$ ) hollow nanolattice when subjected to cyclic uniaxial compression under scanning electron microscopy (SEM), while the corresponding stress-strain curves are shown in Fig. 2c. During the initial stage of compression (Stages I to III), the nanolattice struts mainly deform elastically until they buckle at a plateau stress. At higher compressive strains (Stages III to IV), the nanolattices exhibit localized deformation or buckling at their nodes without any apparent fracture (Fig. 2a) or significant stress drops (Fig. 2c). In this stage, the local strain at the nodes is typically too large for micro/nanolattices with nonductile constituents to withstand, resulting in either catastrophic brittle or localized strut fracture. In contrast, our nanolattice has walls that are significantly more ductile, suppressing otherwise imminent strut fracture. Upon unloading (Fig. 2b), our MEA nanolattices also exhibit large recoverability (over 90\%) and exceptional resilience accentuated by the relatively marginal decrease in energy absorption per unit volume (i.e., toughness) over the course of multiple loading cycles compared to 

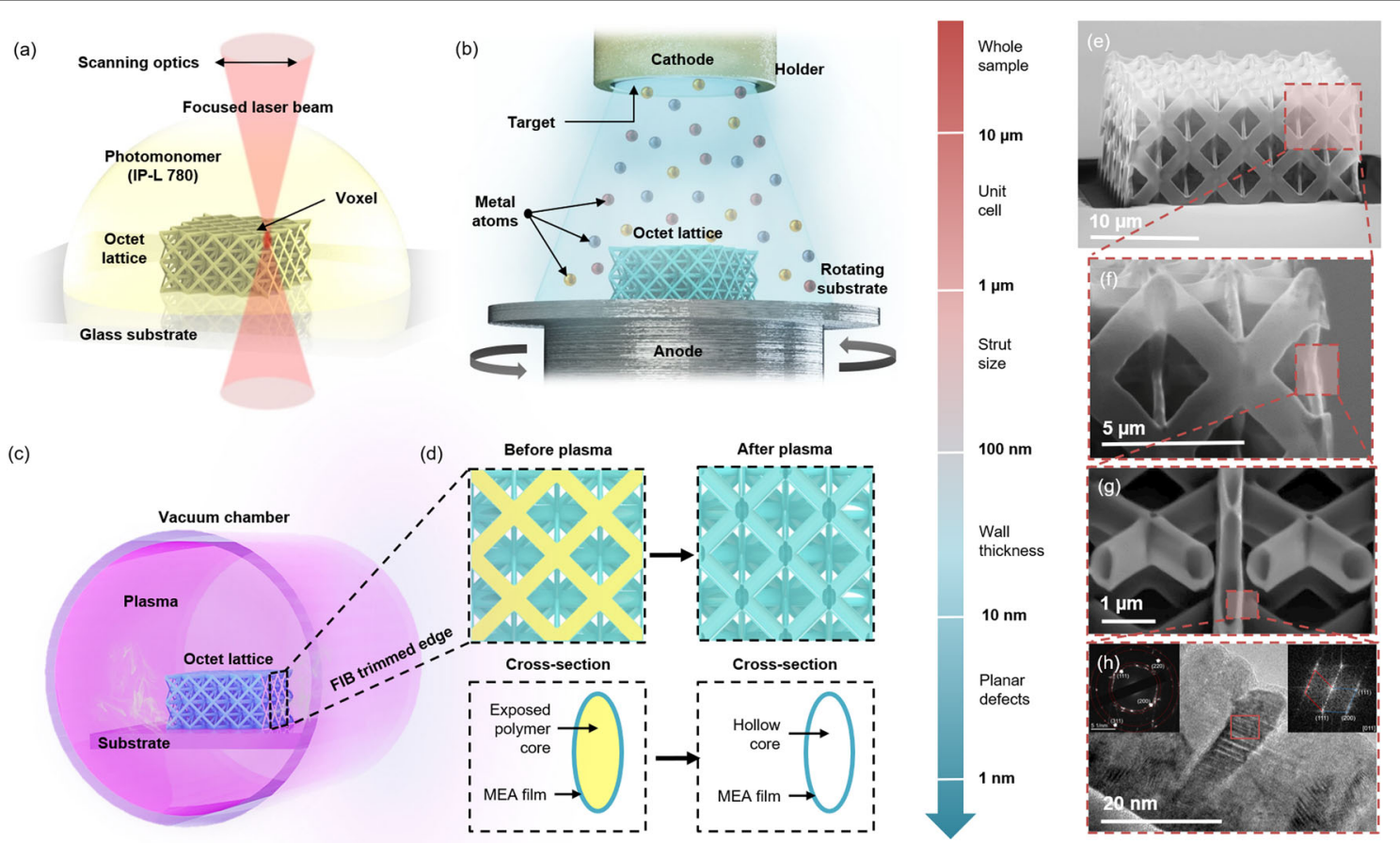

Fig. 1 Fabrication of hollow MEA nanolattices. a Schematic illustration of the two-photon lithography (TPL) technology used to fabricate polymer nanolattices that serve as a physical template for the MEA film. $\mathbf{b}$ Illustration of the DC magnetron sputtering process used to deposit a thin layer of CoCrNi MEA onto the polymer nanolattice scaffold. c Illustration of the octet MEA composite nanolattice during plasma ashing and after focused ion beam milling (to expose the polymer core). $\mathbf{d}$ Schematic depicting the hollow MEA nanolattice produced upon plasma ashing (to remove the polymer core). $\mathbf{e}-\mathbf{h}$ Hierarchical structure of the as-fabricated MEA nanolattices. The insets in $\mathbf{h}$ are the selected area diffraction pattern (SADP) and the corresponding fast Fourier transform (FFT) along the [011] zone axis. The twin boundaries (TBs) are determined to be parallel to one set of the $\{111\}$ planes. Additionally, long streaks appear along two $\{111\}$ planes, indicating the existence of stacking faults (SFs).

previously reported micro/nanolattices, retaining over $60 \%$ of their initial energy in the subsequent cycle (Fig. $2 \mathrm{~d})$. The energy loss coefficient typically refers to the ratio of dissipated energy to the work done during compression, which depicts the hysteresis of a material during cyclic loading ${ }^{21}$. For our MEA nanolattices, the converged energy loss coefficient is greater than 0.8 even after four loading cycles, outperforming previously reported micro/nanolattices ${ }^{1,3,5,21}$.

\section{Deformation mechanism}

The mechanical toughness and resilience of our nanolattices are mainly attributed to the dual elastic and ductile deformation modes that occur in the low- and high-stress regions of the nanolattice, respectively (Fig. 3a-d). The manifestation of this combined deformation mode can be ascribed to two main factors, namely, the external size effect and internal microstructure of the MEA film.

The influence of the size effect can be explained by analyzing the competing failure mechanisms for hollow lattices subjected to uniaxial compression ${ }^{5}$. The transition in deformation modes between shell buckling and fracture can be determined by equating the stresses required to initiate each mechanism, and for elliptical strut lattices with an aspect ratio of approximately 3:1 $(a=3 b)$, the following expression can be used ${ }^{5,29}$ :

$$
\left(\frac{t}{a}\right)_{\text {crit }}=\frac{\sigma_{\text {fracture }}}{E} \sqrt{3\left(1-\nu^{2}\right)}
$$

where $(t / a)_{\text {crit }}$ represents the ratio of the critical wall thickness, $t$, to the major-axis radius of the strut, $a$, at which the shell buckling-to-fracture transition occurs; $\sigma_{\text {fracture }}$ is the fracture strength of the MEA film, which can be taken as $\sigma_{\text {fracture }}=H / 3$, where $H$ is the hardness of the film determined by nanoindentation ${ }^{28,30} . E$ and $v$ represent the Young's modulus and Poisson's ratio of the MEA film, respectively. By using the tested (Fig. S3) and reported values for the CoCrNi film ${ }^{31}, E=214 \mathrm{GPa}, v=0.31$, and $\sigma_{\text {fracture }}=$ $3.4 \mathrm{GPa}$, it can be deduced that $(t / a)_{\text {crit }} \sim 0.026$. The $t / a$ ratio of the thin-walled nanolattices is slightly smaller than the critical value ( 0.025). Therefore, shell buckling, which is usually observed as wrinkling and warping of the hollow struts at the nodes, should dominate over strut fracture. This agrees well with the deformation displayed by the thinwalled nanolattices (Fig. 2a, b), which allows large recoverability to be achieved. Increasing the wall thickness slightly beyond the critical $t / a$ ratio will result in nanolattices with large plastic deformation but low recoverability. For 


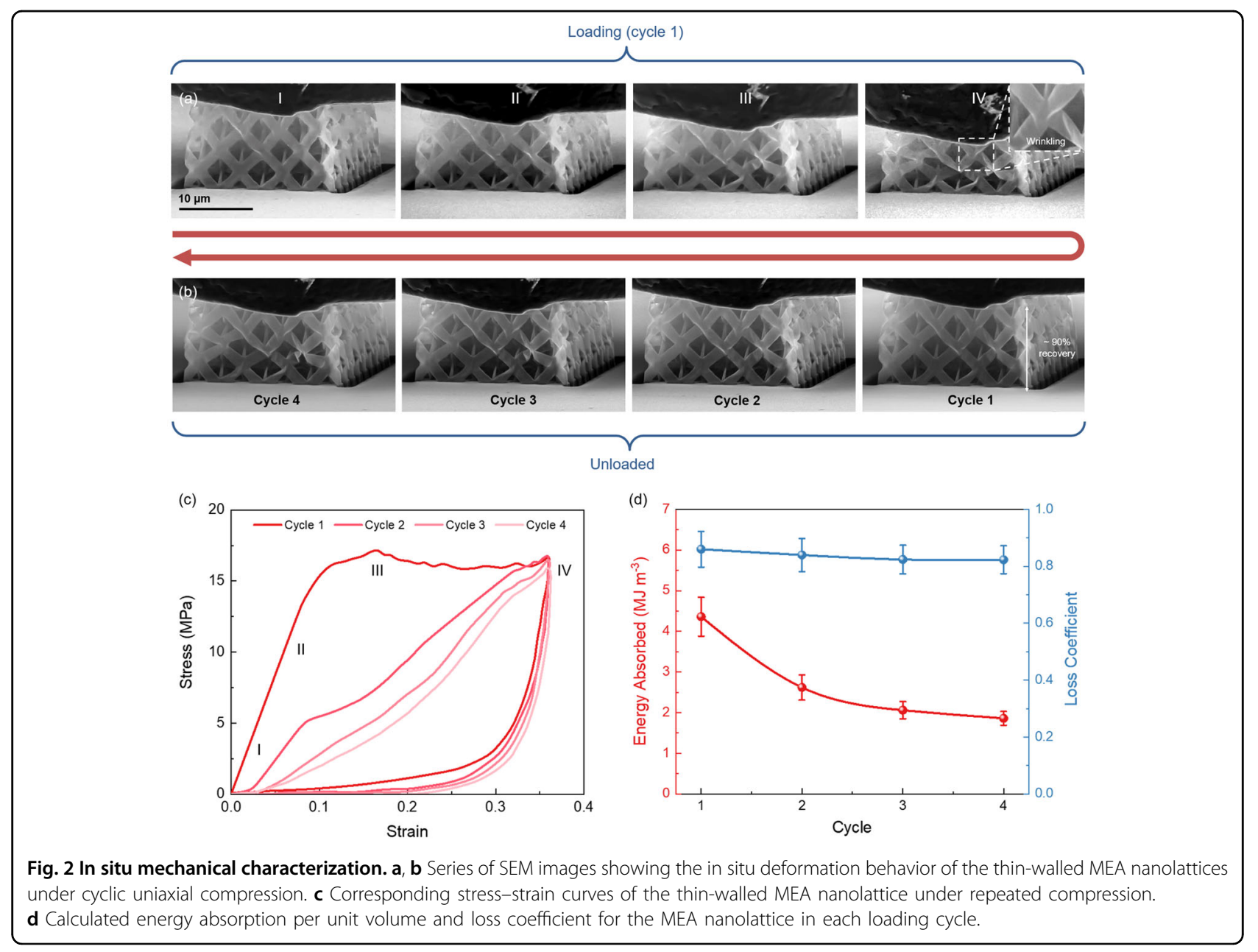

thick-walled nanolattices whose $t / a$ ratios are significantly higher than the critical value, strut fracture dominates, making the nanolattices more brittle (Fig. S4). However, these equations cannot capture the influence of stress concentration and microstructural effects, which has thus far been responsible for localized strut fracture in hollow micro/nanolattices ${ }^{1,5,28,32}$. Intriguingly, localized strut fractures in the high-stress regions were observed to be suppressed in our thin-walled MEA nanolattices, and wrinkled nodes were manifested instead (Fig. 3c). This is mainly ascribed to the inherent ductility of our MEA nanolattices.

The intrinsic deformation mechanism of the $\mathrm{CoCrNi}$ MEA film was investigated via TEM. Figure $1 \mathrm{~h}$ shows a representative TEM image of the MEA film with a thickness of $\sim 30 \mathrm{~nm}$. The MEA grows in a nanosized $(\sim 9.0 \mathrm{~nm})$ columnar grain structure with a face-centered cubic (fcc) phase populated with a high density of nanotwins and SFs. The numerous grain boundaries and SFs serve as significant obstacles to dislocation movement, providing substantial strength by decreasing the dislocation-free pathway $^{33}$. The sub-2-nm-thick twin/SF/matrix lamellar structure of the MEA was revealed by high-resolution TEM observation (Fig. 3f). It has been reported that when the twin boundary spacing is smaller than a critical value, the dense nanotwins in an MEA film could act as detwinning sites, allowing for large plastic deformation to occur $^{34,35}$. Postmortem TEM observation of the MEA film revealed the annihilation of twins in the severely deformed region (Fig. 3e), implying that the ample nanotwins act as detwinning sites, which allows for large plastic deformation to be achieved at the nodes. Therefore, due to the strength and ductility of the MEA film, our thin-walled nanolattices exhibit localized plastic wrinkling in regions of high stress concentration (i.e., nodes) to restrain fracture, while other strut regions show recoverable shell buckling, enabling simultaneous high toughness and resilience with minimal mechanical degradation. The mechanical properties and deformation behavior of the hollow MEA nanolattices are summarized in Table S1.

\section{High specific energy absorption and resilience}

Owing to the high ductility of the MEA film and dual deformation modes, our damage-resistant $\mathrm{CoCrNi}$ hollow 


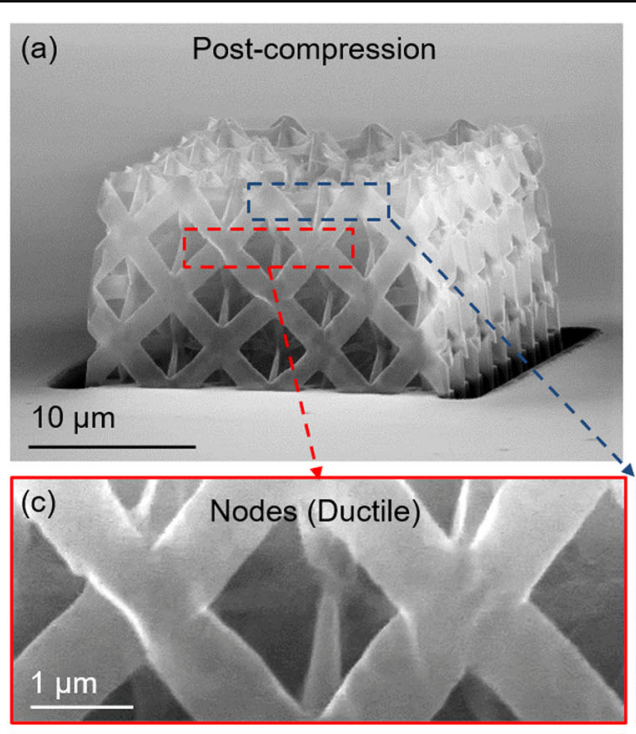

(b) Mises Stress
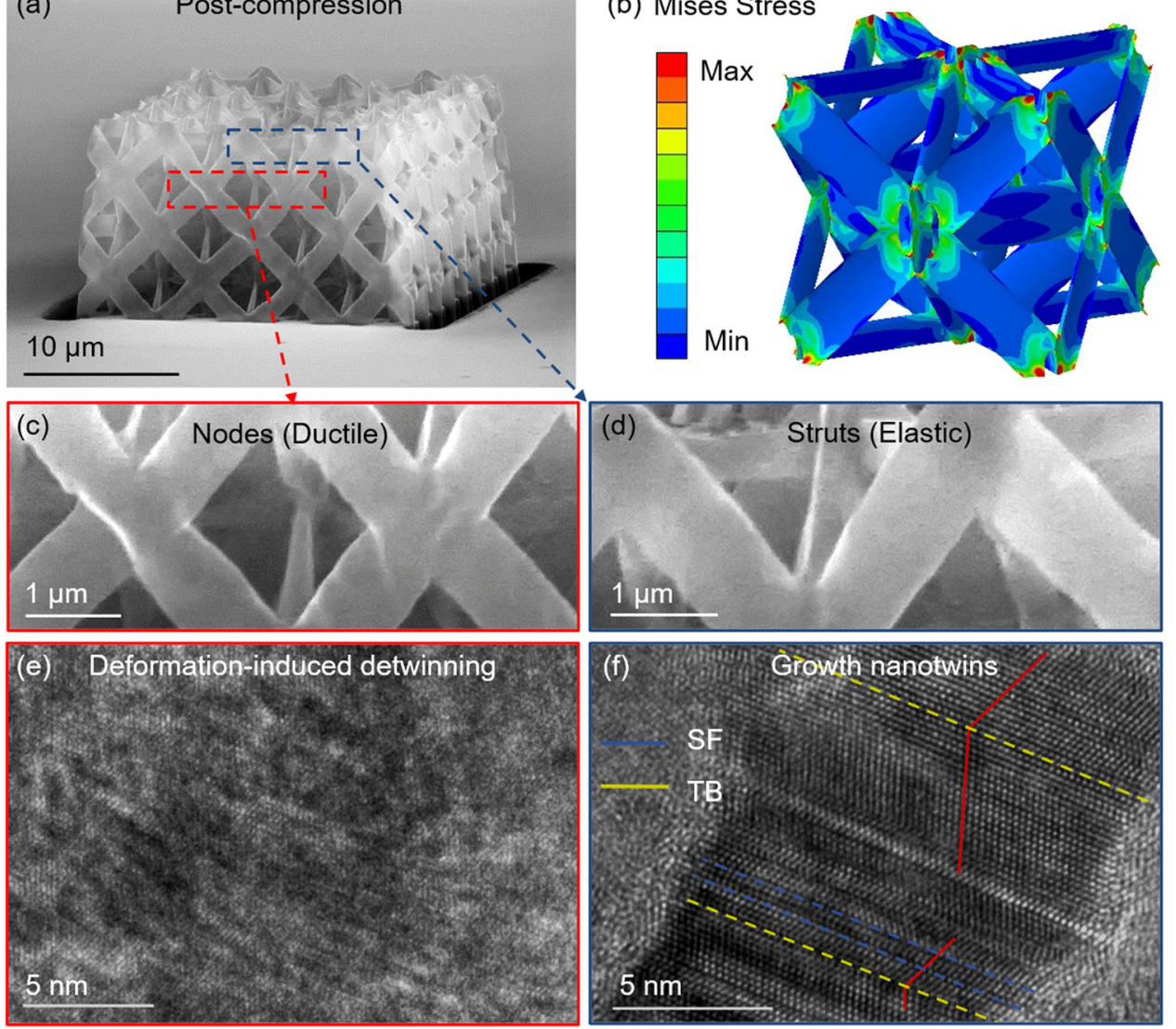

Fig. 3 Microstructural analysis of the post-compression MEA lattice struts. a SEM image of the post-compression MEA nanolattice. $\mathbf{b}$ Finite element analysis (FEA) of the representative hollow MEA octet unit showing the stress concentration regions. c Magnified SEM image showing a plastically deformed node of the nanolattice. $\mathbf{d}$ Magnified SEM image showing the dominant elastic recovery in other areas of the lattice strut. e TEM image showing the detwinned microstructure of the largely deformed MEA film post-compression. $\mathbf{f}$ TEM image of a slightly deformed or elastic region within the CoCrNi MEA film, showing the high density of stacking faults and nanotwins.

nanolattices can exhibit unprecedented specific strength, energy absorption, and resilience compared to previously reported hollow lattices (Fig. 4 and Table S2) ${ }^{1-3,5,28,32,36-38}$. From Fig. 4a, it can be seen that our thin-walled hollow CoCrNi MEA nanolattices possess superior strength (up to $20 \mathrm{MPa}$ ) among all the reported hollow micro/nanolattices while retaining high recoverability $(>90 \%)$. The stretchingdominated octet architecture and high hardness of the $\mathrm{CoCrNi}$ walls are mainly responsible for the exceptional strength of the MEA nanolattices, while the optimized film thickness of the MEA walls allows elastic shell buckling to dominate their deformation behavior, enabling high recoverability to be achieved. Other hollow nanolattices with comparable or higher compressive strength typically exhibit catastrophic or brittle failure at low strains. The specific strength of our thin-walled hollow nanolattices (up to $75 \mathrm{MPa} \mathrm{g}^{-1} \mathrm{~cm}^{3}$ ) is also among the highest reported thus far and even higher than that of previously reported solid-beam HEA microlattices (Fig. S5) ${ }^{39}$. The specific energy absorption (SEA) represents the energy absorption per unit volume divided by the density and is a crucial parameter in determining the efficiency of a material as an energy absorber ${ }^{40}$. The synergy obtained by the employment of the high strength and ductile MEA film with the nanolattice architecture of optimized wall thickness enables our MEA hollow nanolattices to achieve unsurpassed SEA with relatively marginal degradation over multiple loading cycles compared to previously reported lightweight micro/nanolattices (Fig. $4 \mathrm{~b})$. In most reported hollow lattices, localized strut fracture is typically observed upon mechanical loading due to the inherently brittle nature of the load-bearing material. Therefore, in each loading cycle, the accumulated strut fracture continually results in significant deterioration of the mechanical properties. On the other hand, our MEA nanolattices can suppress localized strut fracture due to detwinning in high-stress regions (i.e., nodes). This ultimately enables our nanolattices to preserve more energy in subsequent cycles.

In this work, we demonstrated the fabrication of hollow MEA nanolattices that can attain ultrahigh SEA with 
(a)

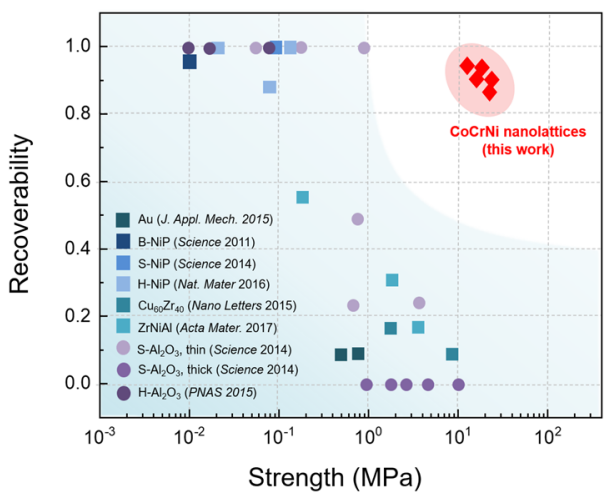

(b)

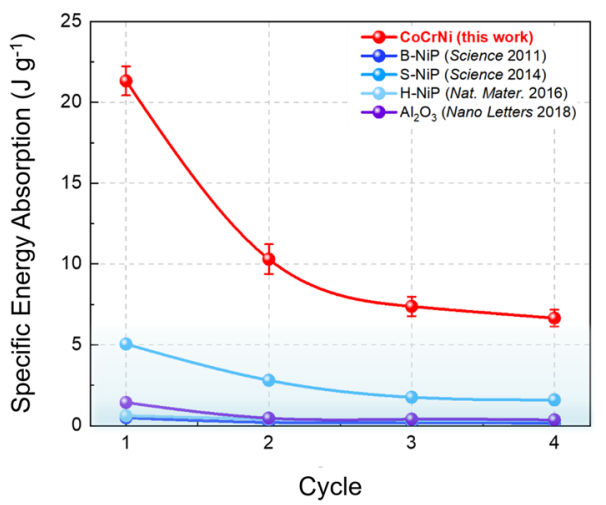

Fig. 4 Performance benchmarking of our MEA nanolattices with some previously reported micro/nanolattice metamaterials. Plot comparing our hollow MEA nanolattices against previously reported lattices in terms of their a recoverability and compressive strength and $\mathbf{b}$ specific energy absorption attained in each loading cycle. The referenced data were extracted from the following: $\mathrm{Au}^{37}, \mathrm{~B}^{\mathrm{N}} \mathrm{NiP}^{1}, \mathrm{~S}_{\mathrm{NiP}} \mathrm{Ni}^{2}, \mathrm{H}-\mathrm{NiP}^{3}, \mathrm{Cu}_{60} \mathrm{Zr}_{40}$ (ref. ${ }^{28}$ ), $\mathrm{ZrNiAl}^{36}, \mathrm{Al}_{2} \mathrm{O}_{3}$ (refs. ${ }^{5,32}$ ), and $\mathrm{H}^{-\mathrm{Al}_{2} \mathrm{O}_{3}}$ (ref. ${ }^{38}$ ).

minimal degradation over multiple loading cycles. This was achieved through the mixed elastic-ductile deformation modes facilitated by the size-induced ductility arising from optimization of the wall thickness and judicious incorporation of dense nanotwins into the low-SF CoCrNi MEA, which act as detwinning sites to suppress localized strut fracture. Coupled with the boundless design space of architected HEAs/MEAs, our findings provide a new path for the creation of ultralight and damage-resistant pure metallic micro/nanolattices with unprecedented combinations of toughness and resilience for next-generation structural and multifunctional applications.

\section{Acknowledgements}

The authors gratefully thank the Shenzhen Science and Technology Innovation Committee for the funding support under grant JCYJ20170413141157573. Part of this project was supported by City University of Hong Kong (Project Nos. 9610461 and 9680108), the Key Research and Development Program of Shaanxi (Program No. 2020GY-252), and the National Natural Science Foundation of China (NSFC) Project 11922215.

\section{Author details}

${ }^{1}$ Department of Mechanical Engineering, City University of Hong Kong, Kowloon, Hong Kong SAR, China. ${ }^{2} \mathrm{CityU}$-Xidian Joint Laboratory of Micro/ Nano-Manufacturing \& Nano-Manufacturing Laboratory (NML), Shenzhen Research Institute of City University of Hong Kong, 518057 Shenzhen, China. ${ }^{3}$ School of Automotive Engineering, Dalian University of Technology, Dalian, Liaoning, China

\section{Conflict of interest}

The authors declare no competing interests.

\section{Publisher's note}

Springer Nature remains neutral with regard to jurisdictional claims in published maps and institutional affiliations.

Supplementary information The online version contains supplementary material available at https://doi.org/10.1038/s41427-021-00306-y.
Received: 18 December 2020 Revised: 24 February 2021 Accepted: 11 March 2021.

Published online: 16 April 2021

\section{References}

1. Schaedler, T. A. et al. Ultralight metallic microlattices. Science 334, 962-965 (2011).

2. Zheng, X. et al. Ultralight, ultrastiff mechanical metamaterials. Science $\mathbf{3 4 4}$ 1373-1377 (2014).

3. Zheng, X. et al. Multiscale metallic metamaterials. Nat. Mater. 15, 1100 (2016).

4. Jang, D., Meza, L. R., Greer, F. \& Greer, J. R. Fabrication and deformation of three-dimensional hollow ceramic nanostructures. Nat. Mater. 12, 893 (2013).

5. Meza, L. R., Das, S. \& Greer, J. R. Strong, lightweight, and recoverable threedimensional ceramic nanolattices. Science 345, 1322-1326 (2014).

6. Bauer, J., Schroer, A., Schwaiger, R. \& Kraft, O. Approaching theoretical strength in glassy carbon nanolattices. Nat. Mater. 15, 438 (2016).

7. Zhang, X., Vyatskikh, A., Gao, H., Greer, J. R. \& Li, X. Lightweight, flaw-tolerant, and ultrastrong nanoarchitected carbon. Proc. Natl Acad. Sci. USA 116 6665-6672 (2019).

8. Bauer, J. et al. Nanolattices: an emerging class of mechanical metamaterials. Adv. Mater. 29, 1701850 (2017).

9. Surjadi, J. U. et al. Mechanical metamaterials and their engineering applications. Adv. Eng. Mater. 21, 1800864 (2019).

10. Schwaiger, R., Meza, L. \& Li, X. The extreme mechanics of micro-and nanoarchitected materials. MRS Bull. 44, 758-765 (2019).

11. Zhang, X., Wang, Y., Ding, B. \& Li, X. Design, fabrication, and mechanics of 3D micro-/nanolattices. Small 16, 1902842 (2020).

12. Bauer, J., Hengsbach, S., Tesari, I., Schwaiger, R. \& Kraft, O. High-strength cellular ceramic composites with 3D microarchitecture. Proc. Natl Acad. Sci. USA 111 2453-2458 (2014).

13. Mieszala, M. et al. Micromechanics of amorphous metal/polymer hybrid structures with 3D cellular architectures: size effects, buckling behavior, and energy absorption capability. Small 13, 1602514 (2017).

14. Eckel, Z. C. et al. Additive manufacturing of polymer-derived ceramics. Science 351, 58-62 (2016).

15. Yeh, J. W. et al. Nanostructured high-entropy alloys with multiple principal elements: novel alloy design concepts and outcomes. Adv. Eng. Mater. 6, 299-303 (2004).

16. Cantor, B., Chang, I., Knight, P. \& Vincent, A. Microstructural development in equiatomic multicomponent alloys. Mater. Sci. Eng. A 375, 213-218 (2004).

17. Tsai, M.H. \& Yeh, J.-W. High-entropy alloys: a critical review. Mater. Res. Lett. 2, 107-123 (2014)

18. George, E. P., Raabe, D. \& Ritchie, R. O. High-entropy alloys. Nat. Rev. Mater. 4, 515-534 (2019). 
19. Gao, L. et al. High-entropy alloy (HEA)-coated nanolattice structures and their mechanical properties. Adv. Eng. Mater. 20, 1700625 (2018).

20. Surjadi, J. U., Gao, L., Cao, K., Fan, R. \& Lu, Y. Mechanical enhancement of coreshell microlattices through high-entropy alloy coating. Sci. Rep. 8, 5442 (2018).

21. Zhang, $X$. et al. Three-dimensional high-entropy alloy-polymer composite nanolattices that overcome the strength-recoverability trade-off. Nano Lett. 18, 4247-4256 (2018).

22. Senkov, O., Wilks, G., Miracle, D., Chuang, C. \& Liaw, P. Refractory high-entropy alloys. Intermetallics 18, 1758-1765 (2010).

23. Gludovatz, B. et al. A fracture-resistant high-entropy alloy for cryogenic applications. Science 345, 1153-1158 (2014).

24. Gludovatz, B. et al. Exceptional damage-tolerance of a medium-entropy alloy CrCoNi at cryogenic temperatures. Nat. Commun. 7, 10602 (2016).

25. Yao, Y. et al. Carbothermal shock synthesis of high-entropy-alloy nanoparticles. Science 359, 1489-1494 (2018).

26. Zhang, N. et al. Lattice oxygen activation enabled by high-valence metal sites for enhanced water oxidation. Nat. Commun. 11, 1-11 (2020).

27. Christensen, J., Kadic, M., Kraft, O. \& Wegener, M. Vibrant times for mechanical metamaterials. MRS Commun. 5, 453-462 (2015).

28. Lee, S.-W., Jafary-Zadeh, M., Chen, D. Z., Zhang, Y.-W. \& Greer, J. R. Size effect suppresses brittle failure in hollow Cu60Zr40 metallic glass nanolattices deformed at cryogenic temperatures. Nano Lett. 15, 5673-5681 (2015).

29. Allen, H. \& Bulson, P. Background to Buckling (McGraw-Hill, 1980).

30. Oliver, W. C. \& Pharr, G. M. An improved technique for determining hardness and elastic modulus using load and displacement sensing indentation experiments. J. Mater. Res. 7, 1564-1583 (1992).
31. Feng, $X$. et al. Heavily twinned CoCrNi medium-entropy alloy with superior strength and crack resistance. Mater. Sci. Eng. A 788, 139591 (2020).

32. Dou, N. G., Jagt, R. A., Portela, C. M., Greer, J. R. \& Minnich, A. J. Ultralow thermal conductivity and mechanical resilience of architected nanolattices. Nano Lett. 18, 4755-4761 (2018).

33. Feng, X., Surjadi, J. U., Li, X. \& Lu, Y. Size dependency in stacking faultmediated ultrahard high-entropy alloy thin films. J. Alloys Compd. 844, 156187 (2020).

34. Li, X., Wei, Y., Lu, L., Lu, K. \& Gao, H. Dislocation nucleation governed softening and maximum strength in nano-twinned metals. Nature 464, 877-880 (2010).

35. Feng, $X$. et al. Effects of nanotwins on the mechanical properties of AlxCoCrFeNi high entropy alloy thin films. Scr. Mater. 139, 71-76 (2017).

36. Liontas, R. \& Greer, J. R. 3D nano-architected metallic glass: size effect suppresses catastrophic failure. Acta Mater. 133, 393-407 (2017).

37. Montemayor, L. \& Greer, J. Mechanical response of hollow metallic nanolattices: combining structural and material size effects. J. Appl. Mech. 82, 071012 (2015).

38. Meza, L. R. et al. Resilient $3 D$ hierarchical architected metamaterials. Proc. Natl Acad. Sci. USA 112, 11502-11507 (2015).

39. Peng, S. et al. Additive manufacturing of three-dimensional (3D)-architected CoCrFeNiMn high-entropy alloy with great energy absorption. Scr. Mater. 190, 46-51 (2021).

40. Tancogne-Dejean, T., Spierings, A. B. \& Mohr, D. Additively-manufactured metallic micro-lattice materials for high specific energy absorption under static and dynamic loading. Acta Mater. 116, 14-28 (2016). 